\title{
A Note on the Symmetry between Bribes and Charges
}

\author{
CHARLES R. PLOTT AND STUART MESTELMAN
}

Purdue University

Lafayette, Indiana 47907

\begin{abstract}
It has been argued that within a dynamic setting, without complete information, the tax and bribe methods of correcting externalities give asymmetrical results. A model is presented which shows that the bribe alternative under such conditions yields optimal results, whereas the tax alternative does not. This conclusion is the opposite of conclusions drawn from other models advanced in the literature on the subject. It cannot be said that within such a setting one policy is better than the other, since all such conclusions depend upon the particular characteristics of each model. (Key words: Quality of water; economics; water management)
\end{abstract}

In their recent article Kamien, Schwartz, and Dolbear [Kamien et al., 1966] produced an example in a dynamic setting, which was intended to establish the 'basic asymmetry between bribes and charges.' ${ }^{1}$ within the context of their model the charge was to be preferred to the bribe, since it would always yield the optimum amount of waste discharge, whereas the bribe would not. By exhibiting a similar model that leads to the opposite conclusion, we show that such short-run asymmetries result from the particular characteristics of each model. Hence one cannot say that the asymmetries resulting from a particular model are 'basic.'

Following along the lines of Kamien et al., we will assume that an upstream firm (firm 1) produces $Q_{1}$ and deposits waste $w$ into the stream. This firm can treat an amount of waste $w-y$, where $y$ is the untreated amount, at a cost of $h(w-y)$. (We shall assume that there is no other way to treat the waste or, equivalently, that all other treatment alternatives are too costly to consider. We shall also assume that $h(w-y)=0$ at $w=y$.) Following the model of Kamien et al., we shall assume that there exists a 'river basin authority' that serves as the agent through which affected parties may

1 The essential featurès of their model have been nicely summarized by A. M. Freeman III, "Bribes and Charges: Some Comments," Water Resources Research, 3, 287-288, 1967. negotiate. Rather than letting the authority serve as a surrogate for all damaged parties as is the case in Kamien et al., we shall assume that there is a single downstream firm (firm 2) producing $Q_{2}$, which is damaged. Firm 2 is assumed to have a nonseparable cost function $C^{2}\left(Q_{2}, y\right)$, where $y$ is the amount of untreated discharge. This is a particularly important deviation from the model of Kamien et al. It means that there is no longer a function $G_{2}(y)$, as in Kamien et al., that relates 'social cost' to untreated waste. Attempts to derive such a function suffer from the classic problems of the allocation of joint costs. A particularly important consequence of this assumption is that 'marginal social cost,' and hence the optimum level of treatment, will depend upon downstream output.

We shall make one more important deviation from Kamien et al. They assume that the amount of waste discharge depends upon the upstream firm output. We shall assume that this is not the case. That is, assume that some activity, necessary for production upstream, causes an amount of discharge $\bar{w}$, and that a change in the level of this activity does not change the level of $Q_{1}$. Several different assumptions about the upstream production process could yield this result. So, whereas Kamien et al. assumed in effect that $Q_{2}$ was constant and $w$ was a variable, we shall assume that $Q_{2}$ is a variable and that $w$ is a constant. 
There is no need to show that in the absence of an institution for exchange firm 1 will undertake no treatment, therefore letting $y=\bar{w}$. The result is the classic problem of externalities. In the spirit of the externalities literature and Kamien et al. in particular, we shall assume that property rights are either given to the upstream firm, in which case firm 2 must bribe firm 1 to treat the waste, or, in case property rights are granted to firm 2, firm 1 must compensate firm 2 by the amount of the damage (the case of the tax). The authority is simply an agent that constructs an institutional means by which the transfer is made and the gains from exchange exhausted.

With perfect knowledge of demands and costs the job of the authority is simple. He simply needs to find

$$
\underset{Q_{2}, y}{\operatorname{Max}_{2}} P_{2} Q_{2}-C^{2}\left(Q_{2}, y\right)-h(\bar{w}-y)
$$

The first-order conditions are

$$
\begin{array}{r}
P_{2}-\left[\partial C^{2}\left(Q_{2}, y\right)\right] /\left(\partial Q_{2}\right)=0 \\
-\left[\partial C^{2}\left(Q_{2}, y\right)\right] / \partial y-[\partial h(\bar{w}-y)] / \partial y=0
\end{array}
$$

so that solving (2) and (3), the optimum $\left(Q_{2}{ }^{0}\right.$, $y^{0}$ ) is found.

If rights lie with the downstream firm, the authority must use the tax alternative. A tax per unit of $y$ equal to $\left[\partial C^{2}\left(Q_{2}{ }^{0}, y^{0}\right)\right] / \partial y$ should be levied on firm 1. The firms will have the following maximum problems. For firm 1 we have

$$
\begin{aligned}
\operatorname{Max}_{Q_{1}, y} & P_{1} Q_{1}-C^{1}\left(Q_{1}\right)-h(\bar{w}-y) \\
- & {\left[\partial C^{2}\left(Q_{2}{ }^{0}, y^{0}\right) / \partial y\right] y }
\end{aligned}
$$

with first-order conditions

$$
\begin{array}{r}
P_{1}-\left[\partial C^{1}\left(Q_{1}\right)\right] / \partial Q_{1}=0 \\
-[\partial h(\bar{w}-y)] / \partial y-\left[\partial C^{2}\left(Q_{2}{ }^{0}, y^{0}\right)\right] / \partial y=0
\end{array}
$$

For firm 2 we have

$\underset{Q_{2}}{\operatorname{Max}} P_{2} Q_{2}-C^{2}\left(Q_{2}, y\right)+\left[\partial C^{2}\left(Q_{2}{ }^{0}, y^{0}\right) / \partial y\right] y$

with first-order condition

$$
P_{2}-\left[\partial C^{2}\left(Q_{2}, y\right)\right] / \partial Q_{2}=0
$$

The solution to the behavioral equations 6 and 8 yields the optimum $Q_{2}{ }^{\circ}, y^{0}$.

Now suppose that $P_{2}$ changed (as opposed to $P_{1}$, which was assumed by Kamien et al.) and the new level $P_{a}$ remains unknown to the authority. A demand function could be substituted in place of $P_{2}$ with no change in the final result. This substitution would make the lack of knowledge assumption more plausible. Or, if imperfect knowledge of demand is not a sufficiently realistic assumption, we could have assumed a change in the form of the cost function $C^{2}\left(Q_{2}\right.$, $y$ ).

At this point we should clarify an additional point. In the Kamien et al. paper the language of welfare economics is not used. They are clearly aware that it could have been used, so for the purpose of being brief we will use it.

If $P_{2}$ changes, and the new level remains unknown to the authority, $Q_{2}^{0}, y^{0}$ are no longer the optimum quantities, and the proper unit tax is no longer $\left[\partial C^{a}\left(Q_{2}{ }^{0}, y^{0}\right)\right] / \partial y$. There are several things that the authority can do, all of which, in general, will yield non-optimum results. We shall consider only one such alternative and indicate some of the problems.

The authority knows the cost functions $C^{2}\left(Q_{2}, y\right)$ and $h(\vec{w}-y)$, so that, if the authority knows the new optimum output of $Q_{2}$, say $Q_{2}{ }^{*}$, it could solve the equation (obtained from (3))

$-\left[\partial C^{2}\left(Q_{2}{ }^{*}, y\right)\right] / \partial y-[\partial h(\bar{w}-y)] / \partial y=0$

to find the optimum output of $y$, say $y^{*}$. It could then use $\left[\partial h\left(\vec{w}-y^{*}\right)\right] / \partial y$ as the proper unit tax on $y$. However, the authority does not know $Q_{2}{ }^{*}$.

Since the authority does not know the optimum $Q_{2}$, it 'might' (following the procedure in Kamien et al.) simply use the current output of the downstream firm to evaluate marginal social costs. The authority would solve (3) to find $y$ as a function of $Q_{2}, y=\Phi\left(Q_{2}\right)$, which indicates for each $Q_{2}$ the amount of $y$ that equates 'marginal damage cost' with 'marginal treatment cost.'

If the authority follows this procedure, firm 1 will

$$
\begin{aligned}
\underset{Q_{1 y}}{\operatorname{Max}_{1}} P_{1} Q_{1}- & C^{1}\left(Q_{1}\right)-h(\bar{w}-y) \\
- & {\left[\frac{\partial C^{2}\left(Q_{2} . \Phi\left(Q_{2}\right)\right)}{\partial \Phi\left(Q_{2}\right)}\right] y }
\end{aligned}
$$


and firm 2 will

$\underset{\mathbf{O}_{2}}{\operatorname{Max}} P_{2} Q_{2}-C^{2}\left(Q_{2}, y\right)+\left[\frac{\partial C^{2}\left(Q_{2}, \Phi\left(Q_{2}\right)\right)}{\partial \Phi\left(Q_{2}\right)}\right] y$

There is no problem with 'gaming' on the part of firm 1, since for each level of $Q_{2}$ the authority knows how much $y$ 'should' exist. In the case the authority has no power to regulate firm behavior, additional problems arise. The situation is that of a nonseparable game, and knowledge of the outcome depends upon the psychology of the players [Davis and Whinston, 1962]. Firm 1's behavioral equations will simply be the first-order condition to (10), which are

$$
\begin{array}{r}
P_{1}-\left[\partial C^{1}\left(Q_{1}\right)\right] / \partial Q_{1}=0 \\
-\frac{\partial h(\bar{w}-y)}{\partial y}-\frac{\partial C^{2}\left(Q_{2}, \Phi\left(Q_{2}\right)\right)}{\partial \Phi\left(Q_{2}\right)}=0
\end{array}
$$

If $Q_{2}$ is optimum, firm 1's behavior assures the optimum level of $y$. But firm 2 can solve (13) to find $y=\psi\left(Q_{2}\right)$. The first-order condition to (11) is therefore

$$
\begin{gathered}
P_{2}-\left[\frac{\partial C^{2}\left(Q_{2}, y\right)}{\partial Q_{2}}+\frac{\partial C^{2}\left(Q_{2}, y\right)}{\partial y} \frac{\partial \psi\left(Q_{2}\right)}{\partial Q_{2}}\right] \\
+\frac{\partial}{\partial Q_{2}}\left[\frac{\partial C^{2}\left(Q_{2}, \Phi\left(Q_{2}\right)\right)}{\partial \Phi\left(Q_{2}\right)} \psi\left(Q_{2}\right)\right]=0
\end{gathered}
$$

which obviously does not yield, in general, the correct amount of $Q_{2}$, and hence the procedure does not yield the correct amount of $y$. Furthermore, closer examination of (14) shows that in this case it is not the payment of revenues to firm 2 alone that causes the deviation from optimum. Even if firm 2 did not receive the tax revenues, the result would be non-optimum. In any case, we can conclude that within the framework of this model the tax alternative does not yield the optimum results.

Now let us examine the case where property rights are given to the upstream firm. Here firm 2 must pay firm 1 to treat the waste. To achieve this result, the authority should charge the downstream firm the cost of waste elimination and let the downstream firm choose the amount of waste it wants eliminated. Firm 2 would

$$
\underset{Q_{a, y}}{\operatorname{Max}_{2}} P_{2} Q_{2}-C\left(Q_{2}, y\right)-h(\bar{w}-y)-A
$$

The first-order conditions

$$
\begin{array}{r}
P_{2}-\left[\partial C^{2}\left(Q_{2}, y\right)\right] / \partial Q_{2}=0 \\
-\left[\partial C^{2}\left(Q_{2}, y\right)\right] / \partial y-[\partial h(\bar{w}-y)] / \partial y=0
\end{array}
$$

are simply the Pareto conditions 5 and 6 . When solved they yield the optimum outputs $Q_{2}^{0}, y^{0}$. Now, the authority offers a bribe to firm 1 of

$g(y)=\left\{\begin{array}{l}A+h(\bar{w}-y) \text { if } y=y^{0} \\ 0 \quad \text { otherwise }\end{array}\right.$

Firm 1 will then maximize

$$
\operatorname{Max}_{Q_{1 y}} P_{1} Q_{1}-C\left(Q_{1}\right)-g(y)-h(\bar{w}-y)
$$

The equilibrium waste output will be the optimum $y=y^{0}$. The constant $A$ assures the authority that firm 1 will participate in the plan.

Assume now that there is a change in $P_{2}$. Firm 2, following (16) and (17), will indicate a new desired level of waste treatment. The authority will change the parameters on $g(y)$ accordingly. The new resulting level of $y$ will be optimum, even though the authority did not know the new $P_{2}$.

The bribe alternative thus yields the same optimum results in the case of imperfect information as it did in the case of perfect information. This was not the case with the tax alternative. In fact, the bribe alternative would remain optimum if the cost function $C^{2}\left(Q_{2}, y\right)$ changed in a manner unknown to the authority. This is not the case with the tax alternative. We have thus established a case of asymmetry completely opposite to that of Kamien et al.

Of course, the model can be changed to yield different results. This is just the point. There are many types of institutional and/or behavioral assumptions that will yield any type of results desired. There appears, therefore, to be no 'basic' asymmetry between bribes and changes, contrary to the assertion of Kamien et al.

\section{REFGRENCES}

Davis and Whinston, Externalities, welfare and the theory of games, J. Political Econ., 241-262, June, 1962.

Freeman, A. M. III, Bribes and charges: Some comments, Water Resources Res., 3, 287-288, 1967.

Kamien, M., N. Schwartz, and F. Dolbear, Asymmetry between bribes and charges, Water Resources Res., 2, 147-157, 1966.

(Manuscript received August 16, 1967.) 